\title{
Convective motion in a vibrated granular layer
}

\author{
A. Garcimartín, ${ }^{*}$ D. Maza, J. L. Ilquimiche, ${ }^{\dagger}$ and I. Zuriguel \\ Departamento de Física, Facultad de Ciencias, Universidad de Navarra, E-31080 Pamplona, Spain
}

(Received 26 September 2001; published 15 February 2002)

\begin{abstract}
Velocity measurements are presented for a vertically shaken granular layer. For frequencies around $110 \mathrm{~Hz}$ and accelerations larger than gravity, the layer develops a convective motion in the form of one or more rolls. The velocity of the grains near the wall has been measured. It grows linearly with the acceleration, then the growth rate slows down. A rescaling with the amplitude of the wall velocity and the height of the granular layer makes all data collapse in a single curve.
\end{abstract}

DOI: 10.1103/PhysRevE.65.031303

PACS number(s): 45.70.Qj, 45.70.Mg

\section{INTRODUCTION}

Granular materials display different behaviors: they can be likened to solids, liquids, or gases [1]. Yet the features and the origin of their behavior are far from understood and are the subject of current research. The focus of this paper is on the motion that arises in a thick layer of noncohesive granular material when submitted to a vertical vibration. Such layers are known to develop large scale collective motions [1-3]. Once a certain acceleration threshold is reached (close to $\mathbf{g}$, the acceleration of gravity) grains begin to move. This movement takes a form similar to convecting fluids heated from below (the Rayleigh-Bénard instability [4]). Other instabilities, such as parametric wave patterns and the formation of a heap, have also been reported for granular layers $[5,6]$. Here we are dealing only with the closed flow of granular material, at velocities much smaller than the maximum speed of the vibrating container. This convective flow is usually downward near the wall, and the grains rise at the center of the container [3]. For a given frequency, if the acceleration is increased there is a bifurcation towards a pattern of rolls [7]. This symmetry-breaking bifurcation is known to display strong hysteresis. Further augmentation of the acceleration leads to a disordered state without any apparent structure.

Some mechanisms have been proposed to explain this phenomenon. The most obvious is the friction of the grains with the walls, as suggested by Knight et al. [3]. Other effect concerns the Reynolds dilatancy notion: a tightly packed granular material must increase its volume if it has to change shape. During each cycle, the material undergoes a process involving a compaction and a dilatation that has been proposed to be at the origin of the motion [8]. And yet another analysis-based on the assumption that a "granular temperature" drives the flow, as in thermal convection-has been recently proposed [9]. The role of the surrounding gas has also been described in the case of the formation of a heap [10]: it acts as a lubricant; but it is generally admitted that it is not at the origin of the motion. Notice that all these expla-

\footnotetext{
*Electronic address: angel@ fisica.unav.es

${ }^{\dagger}$ Permanent address: Laboratorio de Optica Física y Láseres, Facultad de Ciencias Físicas y Matemáticas, Universidad Nacional de La Libertad, Avenida. Juan Pablo II s/n, Trujillo, Peru.
}

nations are not mutually exclusive. While they may all contribute to the convection, it is difficult to ascertain their relative significance in a particular situation.

A measurement of the velocity can clearly help to understand the underlying physics. But carrying out such a measurement in a noninvasive way is complicated. The use of trace particles is possible but painful and time consuming. Magnetic resonance imaging has been employed to obtain velocity profiles along a cross section of the container [3], but the setup is entangled. For quasi-two-dimensional flows, a particle image velocimetry (PIV) technique has been used to put in evidence the convective motion using colored particles [11].We have arranged an experiment that allows us to obtain the local velocities at the wall of the container. In the following section we describe the experiment and the techniques used, then we discuss the results on Sec. III.

\section{EXPERIMENTAL SETUP}

The experimental setup consists of a cylindrical box with the lateral wall and top made of polycarbonate, and the base made of aluminum. The diameter of the box is $52 \mathrm{~mm}$ and the height is $50 \mathrm{~mm}$ (but it is not completely filled). Care was taken to avoid electrostatic effects by sprinkling the box with an antistatic spray and changing the beads whenever they are seen to stick to the wall. In order to rule out any influence from the spray, some runs were carried out in a box of borosilicate glass of the same dimensions without using the antistatic spray; no difference was observed in the measured velocities. The box is attached to the shaft of a shaker. The electromagnetic shaker (TiraVib 52110) is able to deliver a sinusoidal vibration to the box with a distortion smaller than $1 \%$, and the residual transverse acceleration is less than $0.05 \mathrm{~g}$ typically. It is driven by an amplifier that is in turn commanded by a function generator, from where the frequency and amplitude are set. Two accelerometers are attached to the box to measure acceleration in the vertical and one horizontal direction, which is acquired by a digital oscilloscope. Both the function generator and the oscilloscope are linked to a PC.

Several different granular materials have been used. The results we are reporting here have been obtained with glass beads of $0.5 \pm 0.1 \mathrm{~mm}$ in diameter unless otherwise stated. Nevertheless, the same phenomena are also observed in sand, for example, with grains approximately that large but much 
more variable in size and form. Several layer depths were explored in the range $20<N<70$, where $N$ is the layer depth normalized by the bead diameter. In addition, the measurements shown here have been carried out at a frequency $f$ $=110 \mathrm{~Hz}$, although in the range from $80 \mathrm{~Hz}$ to $300 \mathrm{~Hz}$ the behavior was qualitatively similar. The nondimensional acceleration $\Gamma=4 \pi^{2} f^{2} A / g$ (where $A$ is the amplitude) for which measurements were made goes from 0.5 to 7 . For $\Gamma$ $>7$ (this value depends on $\mathrm{N}$ ) the movement becomes rather disordered to make meaningful measurements.

The measurement of the velocities is accomplished with an image acquisition system. It consists of a standard charged coupled device camera with a macro lens. Images are recorded on a commercial VCR for subsequent treatment. The procedure involves the tracking of individual beads. In order to do this, the granular layer is illuminated at an angle, so that each sphere produces a bright spot [Fig. 1(a)]. A spatiotemporal diagram is obtained by registering a vertical line of pixels from the image at regular time intervals and stacking them [Fig. 1(b)]. We perform a morphological image processing to isolate each trace and the local derivative is obtained, which is directly related to the velocity [Fig. 1(c)]. If the traces are obtained in a smaller region where the velocities are approximately constant - by registering a line of pixels shorter than the entire cell, giving almost straight traces - the procedure is much faster: the peaks of a Fourier transform of the image give the velocity, but in this case an averaged velocity is obtained, not a local velocity. Both methods work well if the traces do not depart from the vertical, as is the case in the present experiment. If any bead happens to move briefly in and out of the line that is being registered, it will leave a short trace. With the first method, these traces are filtered out; with the second, they have small significance.

Spatial features of about one tenth of millimeter can be easily detected. The temporal resolution depends on the sampling rate; several hundredths of second are reachable. Nevertheless, unavoidably the beads suffer small displacements in the azimuthal direction due to the rearrangements of the layer, so velocities have a small dispersion. The source of error coming from the dispersion of the velocities adds to the fact that near the bottom the beads leap downward when another bead beneath is absorbed into the bulk. The error of the measurements is mainly statistical, arising from the dispersion of the measured velocities; it amounts to about $10 \%$.

The speed of the beads near the walls depends on the depth [see Fig. 1(c)]. They move faster in the upper zone and their velocity decreases as they approach the bottom, where the grains slow down and are entrained into the bulk-the velocity has a radial component to close the flow. In order to characterize the velocity field, we have measured the velocity in the upper third of the layer, where it is bigger [see Fig. 1(c)]. This velocity can be found in most cases with the second method described above, taking a short line of pixels in that zone. We have checked the consistency of the results in some runs with the first method. As the features of the velocity profile scale homogeneously, the maximal velocity defines the profile, and this figure will be referred to in the following simply as the velocity $\mathbf{v}$. a)
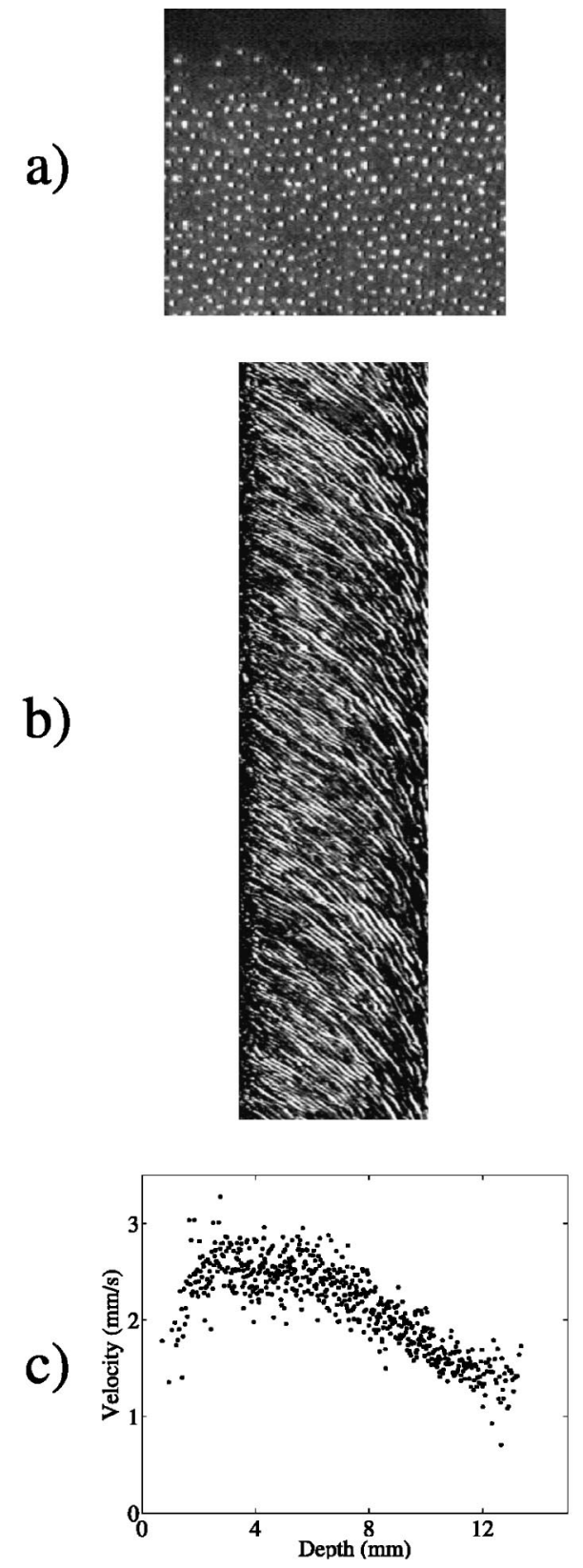

FIG. 1. (a) The beads under side illumination. (b) A spatiotemporal diagram built by stacking a vertical line of pixels sampled at regular time intervals; space corresponds to the horizontal dimension and time to the vertical. Time increases downwards. (c) The velocities obtained from the spatiotemporal diagram shown in (b).

\section{RESULTS AND DISCUSSION}

Let us show the sequence of events for $N=20$ (a similar picture emerges for other layer depths). When $\Gamma$ is small, the beads begin to move uniformly down the wall. Usually a small heap is formed. As $\Gamma$ is increased, the heap decreases in height and by $\Gamma \geqslant 3$ the surface is almost flat. At the same time, the velocity grows larger and larger. At this stage, the velocity near the wall does not depend on the azimuthal position along the wall. The beads go up at the axis and down along the lateral wall, in a toroidal flow.

A further increase of acceleration produces a bifurcation 


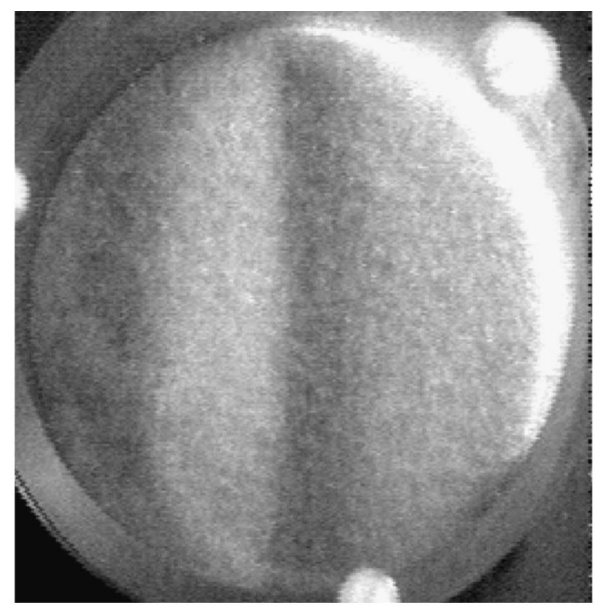

FIG. 2. The surface of the convecting granular material under vertical vibration $(\Gamma=6 \cdot 10, N=20)$. The lateral illumination reveals a pattern of two rolls that protrude slightly at their axes.

towards another pattern: two rolls appear, breaking the circular symmetry of the previous pattern (Fig. 2). The acceleration threshold shows little dependence on the frequency in the range explored; it is about $\Gamma=6$. This transition, as remarked by Aoki and co-workers [7], presents hysteresis: once the rolls have formed, if acceleration is decreased they revert to the previous pattern at a lower value of $\Gamma$. The orientation of the rolls in the circular box from one run to another is seemingly random.

This bifurcation has been described before [12] as a period doubling instability in the vertical motion of the entire layer, although the corresponding values of $\Gamma$ are higher in the present experiment. We have also reproduced some of the standing wave patterns observed by those authors, illuminating the surface with strobed light at a subharmonic fre-

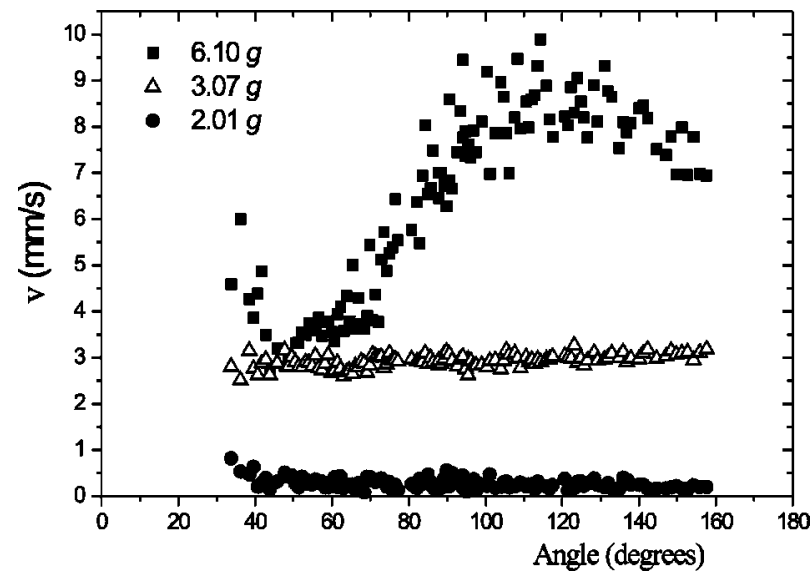

FIG. 3. Velocity profiles near the wall for $N=20$ as a function of the azimuthal angle $\theta$ : circles, $\Gamma=2.01$; triangles, $\Gamma=3.07$; squares, $\Gamma=6.10$. Note that only a portion of the complete circumference is accessible (about $160^{\circ}$ ). All the velocities are downward. It can be seen that $\mathbf{v}$ is constant for small $\Gamma$-the convective pattern is a single toroidal roll-while for larger $\Gamma$ a spatial oscillation is revealed-two rolls are formed, as shown in Fig. 2.

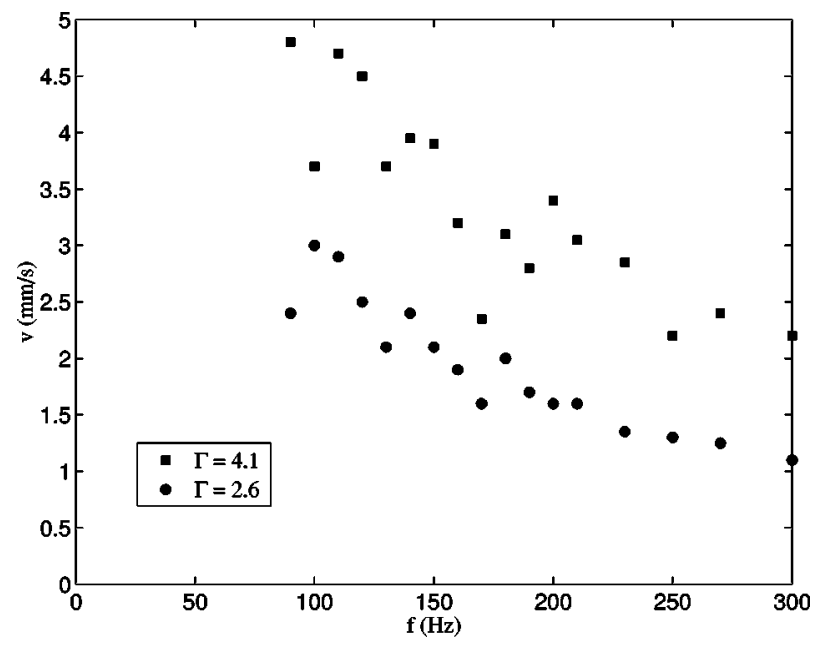

FIG. 4. The frequency dependence of the velocity. Circles correspond to $\Gamma=2.6$ and squares to $\Gamma=4.1$.

quency. Here we deal with the convective motion that affects all the layer, not just the surface.

A profile of the velocities along the azimuthal direction is shown in Fig. 3. For small $\Gamma$ (i.e., $\Gamma=2, \Gamma=3$ ) the downward velocity is constant along the wall, indicating that a toroidal circulation has been established. For $\Gamma=6.10$, however, a modulation is observed superimposed on the downward velocity (note that the mean velocity is not zero). This profile is constant in time. It corresponds to the pattern shown on Fig. 2, which is observed under low angle illumination. Further increase of the acceleration (explored only for some values of $N$ ) leads to an increase in the number of rolls, as described in previous experiments [7], then to a featureless disordered motion.

The resemblance of these spatial patterns with those found in small aspect ratio Bénard convection is striking. When a fluid layer open to the atmosphere heated from below begins to move, fluid goes up at the center and descends near the wall. If the temperature at the bottom is increased, a

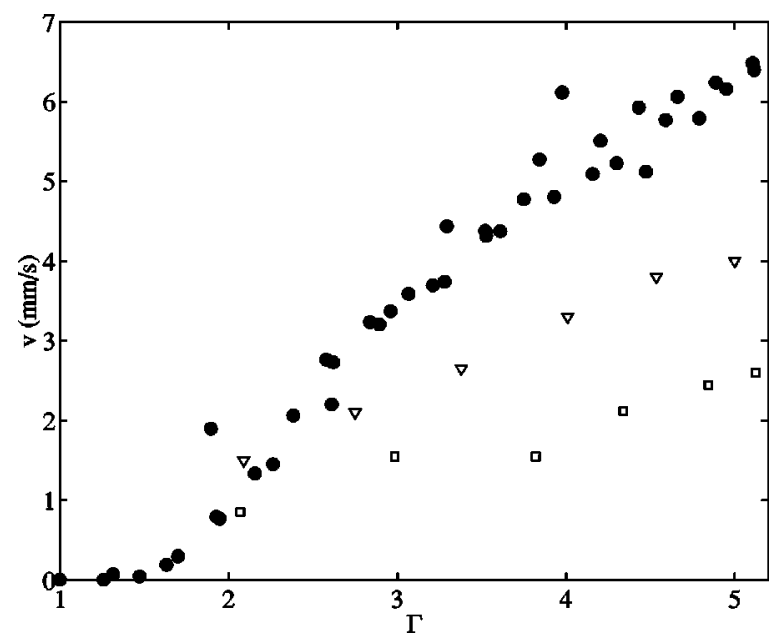

FIG. 5. The velocity vs $\Gamma$ in an evacuated container, for $N$ $=100 / 3$. Squares, 30 mTorr; triangles, 1 Torr; closed circles, atmospheric pressure. 

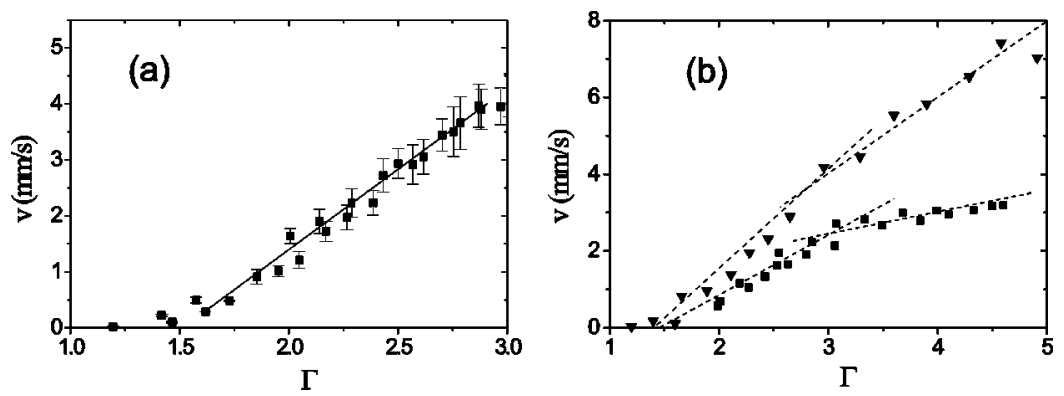

FIG. 6. (a) The dependence of $\mathbf{v}$ on $\Gamma$ hints at an imperfect transcritical bifurcation: a linear growth is apparent (dashed line). (b) For a larger range of accelerations (but still giving a single toroidal convective roll) two linear regimes are seen; in the second one, the slope is smaller. Squares, $N=20$; triangles, $N=40$. Some data points were obtained increasing $\Gamma$, and others decreasing it: no hysteresis is found. The fits are just guides for the eye. pair of rolls appear forming a pattern quite similar to the one found in granular materials [13]. This strongly suggest that the convective motion in a shaken granular layer may be governed by continuum equations with boundary conditions analogous to those of convecting liquids. In order to provide the complete boundary conditions, the velocity field at the bottom of the container is needed. Work is in progress to obtain it. We do not have an explanation, however, for the fact that symmetry along the horizontal midplane is broken: in agreement with [7], for $f=110 \mathrm{~Hz}$ we have observed only downward flow near the wall (but we reproduced an upward flow near the wall, at least locally, for the frequencies and accelerations indicated in Ref. [7]). In fact for a large range of frequencies above $80 \mathrm{~Hz}$ the convective motion can be observed as described here, although the velocity depends on the frequency (Fig. 4).

When the experiment is performed in an evacuated container the velocity decreases substantially. This is shown in Fig. 5, where $\mathbf{v}$ is plotted vs $\Gamma$ for three conditions: at a pressure of 30 mTorr, of 1 Torr, and at atmospheric pressure. The data obtained are in quantitative agreement with the measurements made by Pak et al. [10], and this is valid for other depths we have checked. However, there is one caveat: that paper concerns the formation of a heap, which is partially suppressed in vacuum. We do not claim to recover the scaling law presented by those authors for the case of granular convection. To support it, an extensive survey would be needed.

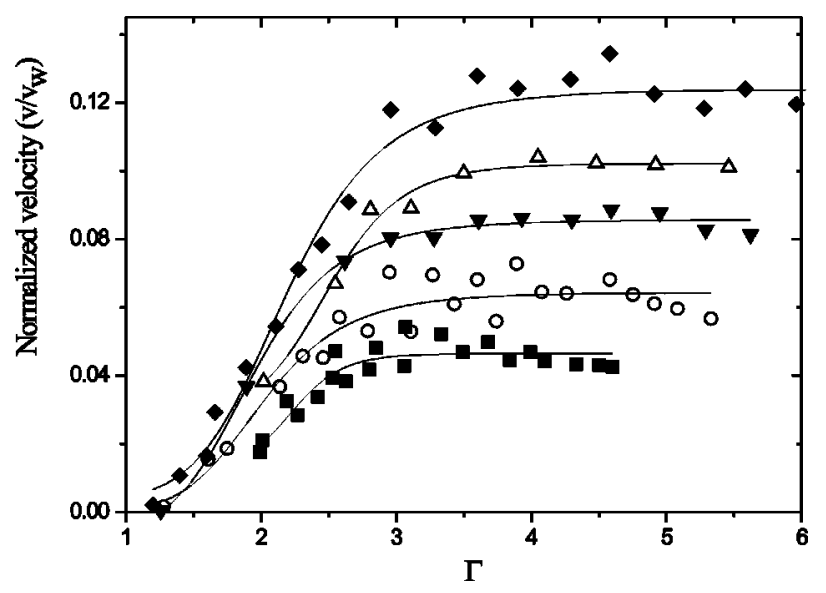

FIG. 7. The normalized velocity $\mathbf{v} / \mathbf{v}_{\mathbf{w}}$ as a function of $\Gamma$. Squares, $N=20$; circles, $N=80 / 3$; down triangles, $N=100 / 3$; up triangles, $N=40$; diamonds, $N=140 / 3$. The fits are guides for the eye.
In order to investigate the dependence of the velocity $\mathbf{v}$ near the wall with the acceleration, we will focus in the first pattern, the one that appears for small $\Gamma(\Gamma<6)$. This planform is concordant with the cross-section profiles obtained by Knight et al. [3]. The velocity field takes the form of a torus, the grains rising at the center and going down near the walls. The dependence of $\mathbf{v}$ on the acceleration is shown in Fig. 6(b) for two layer depths: $N=20$ and $N=40$. The velocity is larger for thicker layers, and it grows monotonically with $\Gamma$. The relationship between $\mathbf{v}$ and $\Gamma$ [a linear growth of $\mathbf{v}$, see Fig. 6(a)] hints at an imperfect transcritical bifurcation. The numerical results of Ramírez et al. [9], who propose a "thermal convection" mechanism, predict a supercritical bifurcation towards their first convective state. Nevertheless, the first convective state they found is banned in the experiment for symmetry reasons (grains do not go up near the wall) and besides their prediction is made for mean values of the velocity.

It is noticeable that before the transition from one to several rolls (for $\Gamma<6$ ) two different regimes exist with two distinct slopes [Fig. 6(b)]. The changing of slope is more evident for the series corresponding to $N=20$. This behavior is more clearly displayed if the velocity $\mathbf{v}$ is normalizeddividing it by the amplitude of the velocity of the container wall $\mathbf{v}_{\mathbf{w}}$. The normalized velocity $\mathbf{v} / \mathbf{v}_{\mathbf{w}}$ is shown in Fig. 7 as a function of $\Gamma$. Clearly all series now give the same behavior: a first stage where $\mathbf{v} / \mathbf{v}_{\mathbf{w}}$ grows with $\Gamma$ and a second one where it remains almost constant. This saturation strongly

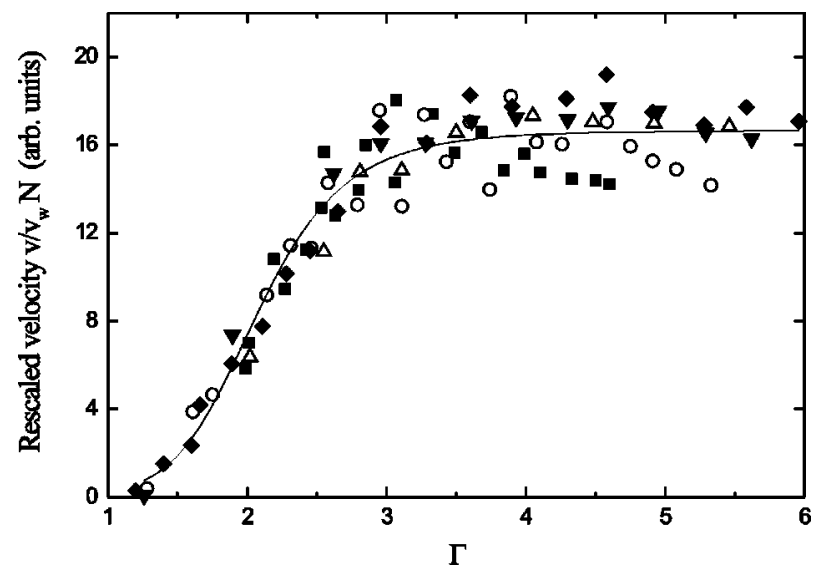

FIG. 8. The rescaled velocity $\mathbf{v} / \mathbf{v}_{\mathbf{w}} \mathbf{N}$ as a function of $\Gamma$. Data are the same as in Fig. 6, all combined. Squares, $N=20$; circles, $N$ $=80 / 3$; down triangles, $N=100 / 3$; up triangles, $N=40$; diamonds, $N=140 / 3$. The fit is a guide for the eye. 


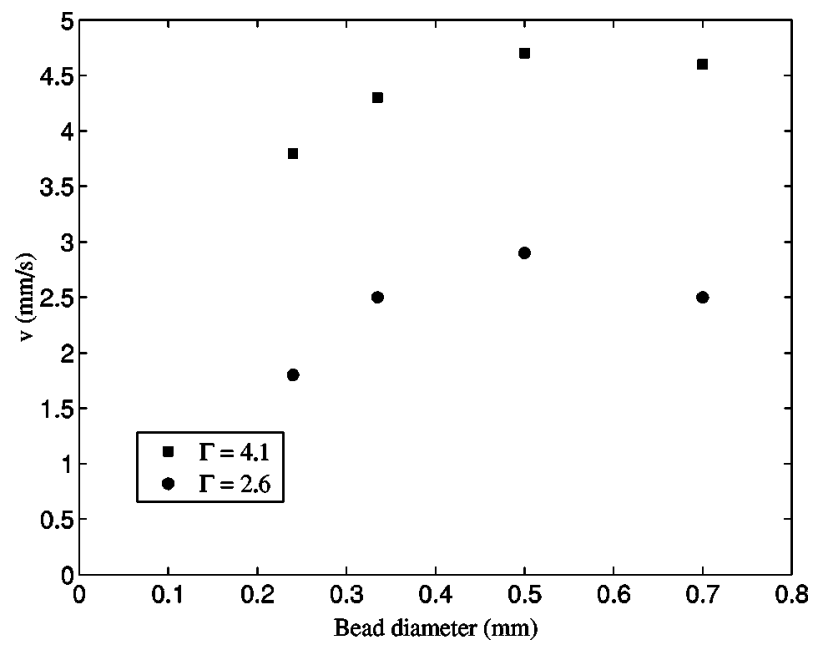

FIG. 9. The convection velocity for several bead diameters, for a fixed layer thickness. Circles correspond to $\Gamma=2.6$ and squares to $\Gamma=4.1$.

suggests an effect related to friction, a force that depends on the velocity, that could either be among the grains themselves or between grains and wall. It is difficult to make an educated guess to decide which is the case; additional work is needed to further clarify this point. The mechanisms invoked by Knight et al. [3] and by Ramírez et al. [9] are both compatible in principle with such a fact.

An additional rescaling can be done dividing the normalized velocity by $N$. After doing this, all the curves collapse in a single one (Fig. 8). This dependency with $N$ is revealing, but again it is difficult to decide whether it is the friction with walls or the "granular temperature" the driving force. The first one should yield a scaling with $N$ for a fixed cross section, as it is the case. The second one could also give a scaling with $N$, although the reason why it should scale linearly is - to our knowledge-unexplained.

We have carried on the experiment in a box of fixed dimensions; in order to assure that the scaling with $N$ is valid we have performed several runs with beads of different diameter. The results are shown in Fig. 9. Other authors [14] had previously found a scaling with the thickness of the layer in several parameters. This is not the case here since the variation of the diameter leaving the layer depth unchanged does have an influence in the convection velocity.

In summary, we have presented the results of an experiment in which the velocity of the grains near the walls has been measured locally. This allows a quantitative description of convective patterns typically associated either to wall drag or to temperature gradients.

\section{ACKNOWLEDGMENTS}

This work was partially funded by Project PIUNA from the Universidad de Navarra, Project No. PB98-0208 from DGYCIT (Spanish Ministerio de Educación) and Project No. HPRN-CT-2000-00158 from the European Community. We thank S. Boccaletti, W. González, H. Mancini, and D. Valladares for their useful comments. J.L.I. acknowledges AECI for financial support, and I.Z. thanks Asociación de Amigos de la Universidad de Navarra for a grant.
[1] H.M. Jaeger and S.R. Nagel, Science 255, 1523 (1992).

[2] J. Duran, Sables, Poudres et Grains (Eyrolles, Paris, 1997).

[3] J.B. Knight, E.E. Ehrichs, V.Y. Kuperman, J.K. Flint, H.M. Jaeger, and S.R. Nagel, Phys. Rev. E 54, 5726 (1996), and references therein.

[4] M.C. Cross and P.C. Hohenberg, Rev. Mod. Phys. 65, 361 (1993).

[5] F. Melo, P. Umbanhowar, and H.L. Swinney, Phys. Rev. Lett. 72, 172 (1994).

[6] C. Laroche, S. Douady, and S. Fauve, J. Phys. (France) 50, 699 (1989); S. Fauve, S. Douady, and C. Laroche, ibid. 50, C3-187 (1989).

[7] K.M. Aoki, T. Akiyama, Y. Maki, and T. Watanabe, Phys. Rev. E 54, 874 (1996).

[8] J. Rajchenbach, Europhys. Lett. 16, 149 (1991); see also E.
Clément and J. Rajchenbach, ibid. 16, 133 (1991).

[9] R. Ramírez, D. Risso, and P. Cordero, Phys. Rev. Lett. 85, 1230 (2000).

[10] H.K. Pak, E. Van Doorn, and R.P. Behringer, Phys. Rev. Lett. 74, 4643 (1995).

[11] R.M. Lueptwo, A. Akonur, and T. Shinbrot, Exp. Fluids 28, 183 (2000).

[12] F. Melo, P.B. Umbanhowar, and H.L. Swinney, Phys. Rev. Lett. 5, 3838 (1995).

[13] T. Ondarçuhu, J. Millán-Rodríguez, H.L. Mancini, A. Garcimartín, and C. Pérez-García, Phys. Rev. E 48, 1051 (1993); M.L. Ramón, D. Maza, and H.L. Mancini, ibid. 60, 4193 (1999).

[14] P.B. Umbanhowar and H.L. Swinney, Physica A 288, 344 (2000). 